\title{
Neurocognitive Disorders in People Living with Human Immunodeficiency Virus Aged 50 Years Old and More in Yalgado Ouédraogo Teaching Hospital
}

\author{
Guira Oumar ${ }^{1,2, *}$, Zombre Yacine ${ }^{2}$, Tieno Herve ${ }^{1,3}$, Drabo Youssouf Joseph ${ }^{1,2}$ \\ ${ }^{1}$ Training and Research Unity in Health Sciences, University Joseph Ki Zerbo, Ouagadougou, Burkina Faso \\ ${ }^{2}$ Internal Medicine Department, Yalgado Ouédraogo Teaching Hospital, Ouagadougou, Burkina Faso \\ ${ }^{3}$ Internal Medicine Department, Bogodogo Teaching Hospital, Ouagadougou, BurkinaFaso
}

Email address:

oumgui@yahoo.fr (G. Oumar), yacinezombre@yahoo.fr (Z. Yacine), tienoh@yahoo.fr (T. Herve), yjdrabo@yahoo.fr (D. Y. Joseph)

${ }^{*}$ Corresponding author

To cite this article:

Guira Oumar, Zombre Yacine, Tieno Herve, DraboYoussouf Joseph. Neurocognitive Disorders in People Living with Human Immunodeficiency Virus Aged 50 Years Old and More in Yalgado Ouédraogo Teaching Hospital. American Journal of Internal Medicine. Vol. 8, No. 4, 2020, pp. 177-181. doi: 10.11648/j.ajim.20200804.16

Received: June 8, 2020; Accepted: July 7, 2020; Published: July 23, 2020

\begin{abstract}
A screening of neurocognitive disorders is required in people living with HIV (PLHIV) to improve their care. We aimed to determine their prevalence, their clinical features and identify their associated factors and their nosology in PLHIV aged 50 years old and more. This cross- sectional study from prospective data was carried out in Yalgado Ouédraogo Teaching Hospital, from February to April, 2019. PLHIV aged 50 years old and more were concerned. Those on antiretroviral therapy for at least 6 months were included. Those having a mental dysfunction that did not allow the use of the questionnaire were not included. Baseline data, those relating to HIV, cognitive and daily living capacities assessment, and the nosology of neurocognitive disorders were collected. Daily living capacities were assessed by the Instrumental Activities of Daily Living score, and the cognitive functions by the Mini Mental State Examination. The Chi-square's or the Fisher's test and the Student's test were used to compare respectively the proportions and the means. The factors for which the p-value was less than 0.20 in a bivariate analysis were included into a logistic regression model for a multivariate analysis with a significance of $\mathrm{p}$ set at $<0.05$. One hundred and two patients were studied: 46 males $(45.1 \%), 56$ females $(54.9 \%)$. The mean age was $57 \pm 5.6$ years. The main antecedent was alcohol consumption (34.3\%). Fifty-nine patients had a nadir of CD4 below $200 \mathrm{cel} / \mu 1$. Eighty one HIV1 patients had an undetectable updated viral load. The mean duration since HIV diagnosis was $147 \pm 62.0$ and that of antiretroviral therapy $130 \pm 50.0$ months. Twenty four $(23.5 \%)$ patients had neurocognitive disorders, particularly in the fields of attention $(100 \%)$ and memory $(87.5 \%)$. A simultaneous disorder in attention, memory and language was the common phenotype (33.3\%). It was Asymptomatic Neurocognitive Impairment (66.6\%), Minor Neurocognitive Disorders (33.3\%). The nosological groups were: "Possible HIV Associated Neurocognitive Disorders (HAND)" (95.83\%), "Probable HAND" (4.17\%), "Certain HAND" and Secondary neurocognitive disorders $(0 \%)$. In a bivariate analysis, age $\geq 65$, male gender, sociocultural status 3 (NSC3), opportunistic infection, the nadir of CD4 were the factors with a p-value $\leq 0.20$. In a multivariate analysis, age $(\mathrm{OR}=4.55)$ and $\mathrm{NSC} 3(\mathrm{OR}=2.55)$ were associated with neurocognitive disorders with respective $\mathrm{p}$-values 0.03 and 0.04. Neurocognitive disorders are not rare in PLHIV aged 50 years old and more in Burkina Faso. However, appropriate assessment tools have to be developed in accordance with the population's socio-cultural specifities.
\end{abstract}

Keywords: Neurocognitive Disorders, HIV, Sub-Saharan Africa

\section{Introduction}

Neurocognitive disorders in people living with Human Immunodeficiency Virus (PLHIV) are acquired, significant and progressive reductions of their capacities in at least two cognitive fields $[1,2]$. Despite the impact of highly active antiretroviral therapies (HAART) on the reduction of neurological damage associated to the severe and chronic 
immunodeficiency [1, 3], neurocognitive disorders remain in PLHIV because of the increase in their life expectancy and the accumulation of cardiovascular risk factors [3-7]. However, a changing in the phenotype of cognitive disorders has been noticed, compared to the profile prior to the advent of HAART $[6,7]$.

Many etiologies, including non HIV-associated conditions and "HIV-associated neurocognitive disorders" (HAND) can cause cognitive impairments in PLHIV. The prevalence of HAND in PLHIV is ranging from less than $50 \%[8,9]$, to 88 $\%$ at a Kenyan Hospital [10].

An early detection of neurocognitive disorders in PLHIV is essential to preserve their autonomy and their socioprofessional integration, as well as to decrease the risk of progression towards dementia and death [4]. Given these benefits, guidelines for screening have been suggested, but there is no consensus about the screening tools and strategies $[3,4]$. For example, the French National Authority for Health recommends a screening for PLHIV aged 50 years old and more, using the Mini Mental State Examination (MMSE) [5]. PLHIV do not benefit during their follow-up from a neurocognitive assessment in Burkina Faso, as in many lowincome countries. We have designed this study to determine the prevalence, describe the clinical features and identify the associated factors and the nosology of neurocognitive disorders in PLHIV aged 50 years old and more in Burkina Faso. The results will be helpful to improve the management of PLHIV.

\section{Material and Method}

\subsection{Design and Setting}

It was a cross-sectional study from a collection of prospective data, carried out from February to April 2019 in the internal medicine department, Yalgado Ouédraogo Teaching Hospital (CHU-YO), Ouagadougou, a reference center for the global management of HIV in Burkina.

\subsection{Patients}

PLHIV aged 50 years old and more, followed-up in the day care hospital, were concerned. Those on HAART for at least 6 months and who gave their verbal consent were included. Those having a mental dysfunction that did not allow the use of the questionnaire were not included.

Patients were identified using the ESTHER Program Evaluation and Operational Monitoring Software (ESOPE). The patients were approached the day of their visit. After explaining the purpose of the study and obtaining their agreement, they were interviewed individually. The interview was in the local language for illiterate patients.

\subsection{Variables}

Baseline data (socio-demographic, antecedent, lifestyle), data relating to the HIV infection (diagnosis, treatment, outcome), the cognitive function and the daily living capacities assessment (tests score), and the nosology of neurocognitive disorders (biological and radiological exams) were collected.

\subsection{Operational Definitions}

Daily living capacities were assessed using the Instrumental Activities of Daily Living (IADL): no impact if score greater than eight, slight impact if less than eight, and significant difficulty if equal zero.

The socio-cultural status (NSC) was classified NSC1: no Certificate of Primary Studies (CEP), NSC2: having the CEP but did not exceeded the $4^{\text {th }}$ year class, NSC3: having the first cycle certificate but did not exceed the terminal class, NSC4: having the Baccalaureate or more [11].

The MMSE was used to evaluate cognitive functions. Neurocognitive disorders were classified as Asymptomatic Neurocognitive Impairment (ANI) face to a decrease of more than one standard deviation in at least two cognitive fields, without an impact on daily living, Minor Neurocognitive Disorders (MND) meeting the criteria of ANI, with an impact on daily living, but without fulfilling the criteria of dementia, and HIV Associated Dementia (HAD) defined as a decrease of at least two standard deviations in at least two cognitive fields, with significant impact on daily living [11].

\subsection{Statistical Analysis}

Data were analyzed with Epi info 7.1.1.14 for the recording, and SPSS2.0 for the analysis. We have compared the group of patients with neurocognitive disorders to the group of patients without neurocognitive disorders in order to determine the associated factors. The Chi-square test or the Fisher's test and the Student's test were used to compare respectively the proportions and the means. The factors for which the p-value was less than 0.20 in a bivariate analysis were included into a logistic regression model for a multivariate analysis with a significance of $p$ value set at $<$ 0.05 .

\subsection{Ethical Considerations}

The study was approved by the internal medicine department responsible. The collection of data has been done in the respect of anonymous and confidentiality, and after obtaining patient's verbal consent.

\section{Results}

\subsection{Socio-demographic Data}

One hundred and two patients were included. Their mean age was $57 \pm 5.6$ years [limits: 50 and 73 ]. They were 46 males $(45.1 \%)$ and 56 females $(54.9 \%)$; the sex ratio was 0.82 . Thirty six patients $(35.2 \%)$ had a NSC1, $23(22.5 \%)$ a NSC2, $28(27.5 \%)$ a NSC3 and $15(14.8 \%)$ a NSC4. The main antecedents were: type 2 diabetes $(4.9 \%)$, hypertension $(23.5 \%)$, psychiatric disease $(1 \%)$, opportunistic infection $(11.8 \%)$, epilepsy $(1 \%)$, cranium-encephalic traumatism (15.7\%), and alcohol consumption (34.3\%). 


\subsection{Data Relating to HIV Infection}

HIV1 was found in 92 patients $(90.2 \%)$ and HIV2 in six patients $(5.8 \%)$. Four patients $(4.0 \%)$ had a double serotype. Eight patients had an updated CD4 level less than $200 \mathrm{cel} / \mu 1$. Fifty-nine had a nadir of CD4 below $200 \mathrm{cel} / \mu 1$. Eighty one HIV1 infected patients had an undetectable updated viral load. The mean duration since HIV infection diagnosis was $147 \pm 62.0$ months [limits: 26 and 323] and that of HAART, $130 \pm 50.0$ months [limits: 26 and 225]. Eighty-nine patients $(87.2 \%)$ were receiving a first line therapy. AZT/3TC/NVP combination was the common regimen $(30.4 \%)$.

\subsection{Features of Neurocognitive Disorders}

\subsubsection{Frequency of Neurocognitive Disorders}

Twenty four $(23.5 \%)$ patients in our study have presented neurocognitive disorders.

\subsubsection{Clinical Profile of Neurocognitive Disorders}

a/ Impairment in cognitive fields

The impairment affected the attention in all the 24 patients $(100 \%)$, followed by memory in 21 patients $(87.5 \%)$. Table 1 shows the frequency of impairment in the different cognitive fields.

Table 1. Frequency of cognitive fields impairments in PLHIV suffering from neurocognitive disorders $(N=24)$.

\begin{tabular}{lll}
\hline Cognitive fields & Total & Frequency \\
\hline Orientation & 03 & 12,5 \\
Learning & 00 & 00 \\
Attention & 24 & 100 \\
Memory & 21 & 87,5 \\
Language & 16 & 66,6 \\
Praxis & 11 & 45,8 \\
\hline
\end{tabular}

b/ Phenotypes of neurocognitive disorders

A simultaneous disorder in the attention, memory and language fields was the most common phenotype (33.3\%). Table 2 shows the distribution of patients according to the phenotypes of the neurocognitive disorders.

Table 2. Distribution of patients according to the phenotypes of their neurocognitive disorders $(N=24)$.

\begin{tabular}{lll}
\hline Phenotypes & Total & Percentage \\
\hline Attention-Memory-Language & 8 & 33.3 \\
Attention-Memory & 7 & 29.2 \\
Attention-Memory-Language-Praxis & 4 & 16.7 \\
Attention-Language-Praxis & 2 & 8.3 \\
Orientation-Attention-Memory-Language-Praxis & 2 & 8.3 \\
Attention-Memory-Praxis & 1 & 4.2 \\
Total & 24 & 100 \\
\hline
\end{tabular}

c/ Severity of neurocognitive disorders

The mean value of the MMSE was 23.12 \pm 4.0 [18-26]. The disorders correspond to ANI in $66.6 \%$ and MND in $33.3 \%$ cases. No case of HAD have been diagnosed.

d/ Impact of neurocognitive disorders on daily living capacities
Sixteen patients $(66.7 \%)$ had no impact on daily living capacities, 8 patients $(33.3 \%)$ had a slight impact and no patient had a significant difficulty.

e/ Nosology of neurocognitive disorders

The biological assessment was carried out in 11 of the 24 patients, and found no abnormality that could explain disorders. Brain Magnetic Resonance Imaging (MRI) was realized only in one patient, and was normal. A "Possible HAND" was the nosological entity in $23(95.83 \%)$, followed by "Probable HAND" in one patient $(4.17 \%)$. No case of "Certain HAND" or Secondary neurocognitive disorders has been diagnosed.

\subsection{Factors Associated with the Neurocognitive Disorders}

\subsubsection{Bi-variate Analysis}

a/ Socio-demographic factors and antecedents associated with neurocognitive disorders

Age $\geq 65$, male gender and NSC3 were the sociodemographic factors associated with neurocognitive disorders with respective p-values $0.03,0.13$ and 0.06 . A history of opportunistic infection was associated with neurocognitive disorders $(\mathrm{p}=0.11)$ (Table 3$)$.

$\mathrm{b} /$ Factors relating to HIV associated with neurocognitive disorders

Table 3. Relation between socio-demographic factors, antecedents and the presence of neurocognitive disorders $(N=102)$.

\begin{tabular}{|c|c|c|c|}
\hline Variable & No disorders & Disorders & P value \\
\hline Age & & & 0.03 \\
\hline$<65$ & $71(79.8 \%)$ & $18(20.2 \%)$ & \\
\hline$\geq 65$ & $7(53.9 \%)$ & $6(46.2 \%)$ & \\
\hline Gender & & & 0.13 \\
\hline Female & $46(82.1 \%)$ & $10(17.9 \%)$ & \\
\hline Male & $32(69.6 \%)$ & $14(29.8 \%)$ & \\
\hline Socio-cultural status & & & 0.06 \\
\hline 1 & $29(80.6 \%)$ & $7(19.4 \%)$ & \\
\hline 2 & $21(91.3 \%)$ & $2(8.7 \%)$ & \\
\hline 3 & $17(60.7 \%)$ & $11(39.3 \%)$ & \\
\hline 4 & $10(71.4 \%)$ & $4(28.6 \%)$ & \\
\hline Diabetes mellitus & & & 0.66 \\
\hline Yes & $4(80.0 \%)$ & $1(20.0 \%)$ & \\
\hline No & $74(76.3 \%)$ & $23(23.7 \%)$ & \\
\hline Hypertension & & & 0.84 \\
\hline Yes & $18(75.0 \%)$ & $6(25.0 \%)$ & \\
\hline No & $60(76.9 \%)$ & $18(23.1 \%)$ & \\
\hline Opportunistic Infection & & & 0.11 \\
\hline Yes & $7(58.3 \%)$ & $5(41.7 \%)$ & \\
\hline No & $71(78.9 \%)$ & $19(21.1 \%)$ & \\
\hline Cranium Traumatism & & & 0.42 \\
\hline Yes & $11(68.8 \%)$ & $5(31.2 \%)$ & \\
\hline No & $67(77.9 \%)$ & $19(22.1 \%)$ & \\
\hline
\end{tabular}

The nadir of CD4 was the factor relating to HIV infection associated with neurocognitive disorders $(\mathrm{p}=0.13)$ (Table 4).

Table 4. Relation between factors relating to HIV infection and the presence of neurocognitive disorders $(N=102)$.

\begin{tabular}{llll}
\hline Variable & No disorders & Disorders & pvalue \\
\hline Serotype & & & 0.55 \\
HIV1 & $71(78 \%)$ & $20(22 \%)$ & \\
HIV2 & $4(66.7 \%)$ & $2(33.3)$ & \\
\hline
\end{tabular}




\begin{tabular}{llll}
\hline Variable & No disorders & Disorders & pvalue \\
\hline $\begin{array}{l}\text { HIV1+2 } \\
\text { NadirofCD4 (cel/ } \mu \text { l) }\end{array}$ & $3(60 \%)$ & $2(40 \%)$ & \\
$<200$ & $48(81.4 \%)$ & $11(18.6 \%)$ & 0.13 \\
$\begin{array}{l}\geq 200 \\
\text { Updated CD4 (cel/ } \mu \text { l) }\end{array}$ & $30(69.8 \%)$ & $13(30.2 \%)$ & \\
$<200$ & $6(75.0 \%)$ & $2(25.0 \%)$ & \\
$\geq 200$ & $72(76.6 \%)$ & $22(23.4 \%)$ & \\
HIV duration (months) & & & 0.60 \\
$\leq 59$ & $6(75.0 \%)$ & $2(25.00 \%)$ & \\
$60-119$ & $33(75.0 \%)$ & $11(25.00 \%)$ & \\
$120-179$ & $17(85.0 \%)$ & $3(15.0 \%)$ & \\
$180-239$ & $22(73.3 \%)$ & $8(26.7 \%)$ & \\
HAART duration (months) & & & 0.69 \\
$\leq 59$ & $6(75 \%)$ & $2(25 \%)$ & \\
$60-119$ & $22(73.3 \%)$ & $8(26.7 \%)$ & \\
$120-179$ & $33(75 \%)$ & $11(25 \%)$ & \\
$180-239$ & $17(85 \%)$ & $3(15 \%)$ & \\
\hline
\end{tabular}

\subsubsection{Multivariate Analysis}

Only the age and NSC3 remain associated with the neurocognitive disorders with respective p-values 0.03 and 0.04 . Thus, patients aged $\geq 65$ years old and those with a NSC3 were more likely to have neurocognitive disorders $(\mathrm{OR}=4.55$ and 2.55 respectively). Table 5 represents the factors associated with the neurocognitive disorders in a multivariate analysis.

Table 5. Multivariate analysis of factors associated with the neurocognitive disorders in PLHIV aged 50 years old and more.

\begin{tabular}{llll}
\hline Co-variables & Odd Ratio & IC $_{\mathbf{9 5} \%}$ & P value \\
\hline Age & 4.55 & {$[1.15-17.90]$} & 0.03 \\
Socio-cultural status & & & \\
1 & 1 & - & - \\
2 & 0.36 & {$[0.06-2.06]$} & 0.25 \\
3 & 2.55 & {$[0.76-8.46]$} & 0.04 \\
4 & 1.58 & {$[0.35-7.06]$} & 0.54 \\
Opportunistic Infection & 3.18 & {$[0.75-13.47]$} & 0.11 \\
Nadir of CD4+ & 2.70 & {$[0.93-7.79]$} & 0.06 \\
\hline
\end{tabular}

\section{Discussion}

The study was designed to determine the prevalence, describe the clinical features and identify the associated factors and the nosology of neurocognitive disorders in PLHIV aged 50 years old and more in Burkina Faso. About the methodological proceedings, the study had limitations relating to the cognitive functions and the daily living capacities assessment tools, which contents are not always appropriate for the majority of the local populations; there is no-validated tool for them. In a recent review, Mwangala reported the lack of a standardized and contextually HAND screening tools in Sub Saharan Africa, and pointed a need for further validation of existing tools and development of new HAND screening tools in Sub Saharan Africa [12]. In addition, the charges of biological and radiological assessment did not allow the complete etiological assessment of neurocognitive disorders for most patients in low-income countries.

The frequency of neurocognitive disorders is found to be $23.5 \%$. This is within the interval $6 \%$ to $69 \%$ reported by some authors $[13,14]$. In Sub Saharan Africa, the prevalence reaches $88 \%$ in Kenya [10], 58, 23\% in Uganda [14] and 49, $7 \%$ in Zimbabwe [16]. The differences could result of the variability in the studies populations, the assessment tools and strategies, and the regional distribution of the neuropathogenic clade D of HIV having a higher potential of causing HAND $[17,18]$.

Two thirds and one third patients with neurocognitive disorders had respectively ANI and MND. No HAD was noticed. This corroborates 98\% ANI and MND and 2\% HAD reported in Kenya [10]. The clinical profile of HAND has changed, compared to the pre-HAART era, the severe forms having decreased with the scale up of HAART. On the other hand, cognitive impairments in PLHIV remain, because of an increase of ANI and MND [6, 7, 19].

The predominant prevalence of the moderate forms of neurocognitive disorders could explain the clinical features, characterized mainly by the impairment in the domains attention and memory. A similar observation was found in Zimbabwe [16]. The disorders in attention, concentration and memory, meaning a subcortical dysfunction, are known to be the initial symptoms at the occurring of neurocognitive disorders. Also, subcortical symptom became the main clinical expression of neurocognitive dysfunction in PLHIV in the HAART era [7, 20]. These impairments could potentially impact the adherence to care, the autonomy in daily living, and the socio-professional insertion of patients.

The antecedents and co-morbidities of patients are similar to those mentioned in the literature, except the co-infection HIV-Hepatitis C [18]. The absence of this co-infection could be explained by its lower frequency in the elderly, as reported by authors in Europe and Burkina [20, 21].

Possible HAND was the main nosological group of neurocognitive disorders. However, this finding must be interpreted, taking into account the limits in accessing to the etiological assessments, particularly the absence of brain MRI in almost all patients (even if the normality of MRI does not exclude the diagnosis of HAND).

In accordance with the literature, elderly was found to be a factor associated with neurocognitive disorders [9, 13, 15]. Other factors such as the gender, the nadir of CD4, the coinfection HIV-Hepatitis $\mathrm{C}$ are reported as risk factors of neurocognitive disorders, but were not found in our study. On the other hand, NSC3 was found to be a factor associated with neurocognitive disorders in our study, everything not having been reported previously, for example in Kenya and Zimbabwe [10, 16]. Further analysis could be helpful to explain this result. We also found, like some authors, that the duration of the infection is not a factor associated with neurocognitive disorders [20].

\section{Conclusion}

Neurocognitive disorders are not rare in PLHIV aged 50 years old and more in Burkina Faso. The clinical feature corresponds to the moderate cognitive impairments, affecting particularly the domains attention and memory. Elderly and 
the high socio-cultural status are the factors associated with neurocognitive disorders. The impairment in different cognitive fields, beyond the risk of therapeutic noncompliance, could decline patient's capacities in their daily living, suggesting why the importance of the early screening and treatment in accordance with international recommendations. However, appropriate assessment tools have to be developed in accordance with the socio-cultural specificities of the local populations in order to have a reliable prevalence of the disorders in PLHIV.

\section{Conflict of Interests}

The authors declare that there is no conflict of interest in relation to the content of the manuscript.

\section{References}

[1] https://www.has-sante.fr. Haute Autorité de Santé. Parcours de soins des patients présentant un trouble neurocognitif associé à la maladie d'Alzheimer ou à une maladie apparentée.

[2] Smail R. C, Brew B. J. HIV-associated Neurocognitive Disorder. Handb Clin Neurol 2018; 152: 75-97.

[3] Mc Arthur JC, Steiner J, Sachtor Netal. Human immune deficiency virus-associated neurocognitive disorder: Mind the gap. Ann Neurol 2010; 67 (6): 699-714.

[4] Moulinier A. Manifestations neurologiques. In Girard P-M, Katlama C, Pialoux G. VIH. Paris: doin; 2011: p 126-130.

[5] https://www.has-sante.fr. Haute Autorité de Santé. VIH. Consultation de suivi en médicine générale des personnes sous traitement antirétroviral.

[6] Sacktor N. Changing Clinical Phenotypes of HIV-Associated Neurocognitive Disorders. J Neurovirol 2018; 24 (2): 141-145

[7] Eggers C, Arendt G, Hahn K, Husstedt I. W, Maschke M, Neuen-Jacob E, and al. HIV-1-associated neurocognitive disorder: epidemiology, pathogenesis, diagnosis, and treatment. J Neurol 2017; 264: 1715-1727.

[8] Bonnet F, Amieva $\mathrm{H}$, Marquant $\mathrm{F}$ et al. Cognitive disorders in HIV-infected patients: are they HIV-related? AIDS 2013; 27: 391-400.

[9] Geny C. Troubles cognitifs et infection par le virus de l'immunodéficience humaine. Lettre de l'infectiologue 2012; XXVII (5): 198-204.
[10] Mugendi A. G, Kubo M. N, Nyamu D. G, Mwaniki L. M, Wahome S. K, Haberer J. E. Prevalence and Correlates of Neurocognitive Disorders among HIV Patients on Antiretroviral Therapy at a Kenyan Hospital. Neurology Research International 2019; 1-10.

[11] Kalafat M, Hugonot Diener L, Poitrenaud J. Standardisation et étalonnage français du Mini Mental State, version GRECO. Revue de Neuropsychologie 2003; 13 (2): 209-236.

[12] Mwangala P. N, Newton C. R, Abas M, Abubakar A. Screening tools for HIV-associated neurocognitive disorders among adults living with HIV in sub-Saharan Africa: A scoping review. AAS Open Research 2019, 1: 28: 1-22

[13] Lescure F X, Moulignier A. Troubles cognitifs associés à l'infection VIH. Journal des Anti-infectieux 2014; 16 (2): 6473.

[14] Vivithanaporn P, Heo G, Gamble Jetal. Neurologic disease burden in treated HIV/AIDS predicts survival: a populationbased study. Neurology2010; 75: 1150-8.

[15] Namagga J. K. Prevalence and Risk Factors of HIVAssociated Neurocognitive Disorders in Rural Southwestern Uganda. J Assoc Nurses AIDS Care 2019; 30 (5): 531-538.

[16] Nyamayaro P, Gouse H, Hakim J, Robbins R. N, Chibanda D. Neurocognitive impairment in treatment experienced adults living with HIV attending primary care clinics in Zimbabwe. BMC Infectious Diseases 2020; 20: 383: 1-20.

[17] Yusuf J, Hassan A, Mamman A. I, Muktar H. M, Suleiman A. M, Baiyewu O. Prevalence of HIV-associated neurocognitive disorder (HAND) among patients attending a tertiary health facility in northern Nigeria. Journal of the International Association of Providers of AIDS Care (JIAPAC) 2017; 16 (1): 48-55.

[18] Letendre S. Central nervous system complications in HIV disease: HIV-associated neurocognitive disorder. Top Antivir Med 2011; 19: 137-42.

[19] Barukh K. Dépistage et prise en charge des troubles cognitifs liés au VIH : évaluation de la filière EVACOG. Thèse de doctorat en médecine, université Paris Diderot-Paris7; 2014. P 71 .

[20] Moulignier A. Le complexe démentiel associé au VIH : aspects particuliers chez les sujets âgés. Psychol Neuropsychiatr vieil 2007; 5 (3): 193-207.

[21] www.anrs.fr. Méda N, Tuaillon E, Kania Detal. Hepatitis B and $\mathrm{C}$ seroprevalence, Burkina Faso: across-sectional study. 